\title{
SQL Server Versions in Distribution, Parallelism and Big Data
}

\author{
Rahmat Zolfaghari \\ Islamic Azad University, \\ Hashtgerd Branch, \\ Department of Computer Engineering,
}

Tehran, Iran

\section{ABSTRACT}

Microsoft SQL Server is a relational database management system developed by Microsoft. As a database server, it is a software product with the primary function of storing and retrieving data as requested by other software applications which may run either on the same computer or on another computer across a network (including the Internet). Microsoft markets at least a dozen different editions of Microsoft SQL Server, aimed at different audiences and for workloads ranging from small single-machine applications to large Internet-facing applications with many concurrent users. In
\end{abstract}

1988 Microsoft joined Ashton-Tate and Sybase to create a variant of Sybase SQL Server for IBM OS/2 (then developed jointly with Microsoft), which was released the following year(2000 -2016). According the role of Distribution, Parallelism and Big Data in Microsoft SQL Server specifically reviewed.

\section{Keywords}

Distribution, Parallelism, Big Data, SQL Server

\section{INTRODUCTION}

Table 1. SQL Server release history [2]

\begin{tabular}{|l|l|l|l|l|}
\hline \multicolumn{1}{|c|}{ Version } & Year & \multicolumn{1}{|c|}{ Release name } & \multicolumn{1}{c|}{ Code name } & Internal version \\
\hline 8.0 & 2000 & SQL Server 2000 & Shiloh & 539 \\
\hline 8.0 & 2003 & SQL Server 2000 64-bit Edition & Liberty & 539 \\
\hline 9.0 & 2005 & SQL Server 2005 & Yukon & $611 / 612$ \\
\hline 10.0 & 2008 & SQL Server 2008 & Katmai & 661 \\
\hline 10.25 & 2010 & Azure SQL DB & Cloud Database or Cloud DB & - \\
\hline 10.50 & 2010 & SQL Server 2008 R2 & Kilimanjaro (aka KJ) & 665 \\
\hline 11.0 & 2012 & SQL Server 2012 & Denali & 706 \\
\hline $\mathbf{1 2 . 0}$ & 2014 & SQL Server 2014 & SQL14 & 782 \\
\hline 13.0 & 2016 & SQL Server 2016 & - & 852 \\
\hline
\end{tabular}

Old version $\square$

Older version, still supported

Latest version

Latest preview version

Table 2. SQL Server Basic Features[3]

Database Engine, Analysis Services, Integration Services, Replication, Reporting Services, Notification Services, Service Broker, Full-Text Search, Master Data Services, SharePoint Integration, Data Quality Services, R Services.

Table 3. SQL Server Versions Features[4]

\begin{tabular}{|c|c|}
\hline Version & Features \\
\hline SQL Server 2000 & $\begin{array}{l}\text { Enterprise Manager, Query Analyzer, SQL Profiler, Service Manager, Data } \\
\text { Transformation Services (DTS), XML and HTTP Support, Book Online }\end{array}$ \\
\hline SQL Server 2005 & $\begin{array}{ccc}\text { Relation } & \text { Database, Replication } & \text { Services, Analysis Services, Reporting } \\
\text { Services } & \text {,Management } & \text { Tools } \\
& & \text {,Development Tools, SSIS, Business } \\
& \text { Intelligence, new method for database programing }\end{array}$ \\
\hline
\end{tabular}




\begin{tabular}{|c|c|}
\hline SQL Server 2008 & $\begin{array}{l}\text { Support for Up to } 256 \text { Processor Cores, Power Pivot and Self-Service BI, Multi } \\
\text { server Management, Master Data Services, Stream Insight, Report Builder 3.0, } \\
\text {.NET Framework Support, Hyper-Threading Support, Snap shot and Mirror } \\
\text { Support for Database }\end{array}$ \\
\hline SQL Server 2008 R2 & $\begin{array}{l}\text { Analysis Services, Multidimensional Data, Analysis Services, Data Mining, } \\
\text { Integration Services, Master Data Services, Replication, Reporting Services, } \\
\text { SharePoint Integration, Service Broker }\end{array}$ \\
\hline SQL Server 2012 & $\begin{array}{l}\text { Always On Availability Groups, Windows Server Core Support, Column store } \\
\text { Indexes, User-Defined Server Roles, Enhanced Auditing Features, BI Semantic } \\
\text { Model, Sequence Objects, Enhanced PowerShell Support, Distributed Replay, } \\
\text { Power View, SQL Azure Enhancements, Big Data Support. }\end{array}$ \\
\hline SQL Server 2014 & $\begin{array}{l}\text { New In-memory OLTP Engine, Enhanced Windows Server } 2012 \text { Integration, } \\
\text { Enhanced to Always on Availability Groups, Enhancements to Backups, } \\
\text { Updateable Column store Indexes, SQL Server Data Tools for Business } \\
\text { Intelligence (BI), Power BI For Office } 365 \text { Integration }\end{array}$ \\
\hline SQL Server 2016 & $\begin{array}{l}\text { Always Encrypted, Dynamic Data Masking, JSON Support, Multiple Temp OB } \\
\text { Database Files, Poly Base, R comes to SQL Server, Query Store, Row Level } \\
\text { Security, Stretch Database, Temporal Table }\end{array}$ \\
\hline
\end{tabular}

\section{DIFFERENCES, SQL SERVER VERSIONS FEATURES [5]}

Table 4. Difference between SQL server 2000 and 2005

\begin{tabular}{|l|l|}
\hline SQL Server 2000 & SQL Server 2005 \\
\hline 1.Quary Analyser and Enterprise manager are separate & A. Both are combined as SSMS \\
\hline 2.No XML data type is introduced & B. XML data type is introduced \\
\hline 3.We can create maximum of 65,535 database & C. We can create 2(pow(20)) -1 database \\
\hline 4. Exception Handling is not here & D. Exception Handling is there \\
\hline 5. can,t compress the tables and indexes & E. can compress the tables and indexes \\
\hline 6. Determine data type is used for both date and time & F. Determine is used for both date and time \\
\hline 7.No var char(max) or var binary(max) is available & G. var char(max) or var binary(max) is available \\
\hline 8. No SSIS is included & H. SSIS is started using \\
\hline 9. PIVOT and UNPIVOT functions are not used & I. PIVOT and UNPIVOT functions are used \\
\hline 10.Cant bulk copy update & J. Cant copy update \\
\hline 11. can,t Encrypt the Database & K. can Encrypt the Database \\
\hline
\end{tabular}

Table 5. Difference between Sql server 2005 and 2008

\begin{tabular}{|l|l|}
\hline SQL Server 2005 & SQL Server 2008 \\
\hline 1.We can,t able to Encrypt the entric Database & A. We able to Encrypt the entric Database \\
\hline 2.Does n,t provide Backup Encryption & B. Introduced Backup Encryption \\
\hline 3.XML Datatype is introduced & C. XML Datatype is implemented and used \\
\hline 4. FILESTREAM is not there & D.FILESTREAM is introduced \\
\hline 5. LINQ is not there & E. LINQ is introduced for retrieving multiple type of data \\
\hline 6. Table valued parameters is not there in sql 2005 & F. Table valued parameters is introduced in sql 2005 \\
\hline
\end{tabular}




\begin{tabular}{|l|l|}
\hline 7.No varchar(max) orvarbinary(max) is available & G. varchar(max) orvarbinary(max) is available \\
\hline 8. Data synchronization isn,t introduced & H. 8. Data synchronization introduced \\
\hline
\end{tabular}

Table 6. Difference between Sql server 2008 and 2008R2

\begin{tabular}{|l|l|}
\hline SQL Server $\mathbf{2 0 0 8}$ & SQL Server 2008R2 \\
\hline 1.It support maximum of 64 logical process & A. It support maximum of 256 logical process \\
\hline 2.MDS part of BI is not included in SQL Server 2008 & B. MDS part of BI is included in SQL Server 2008R2 \\
\hline 3.Poverpivot in BI isn,t implemented in SQL Server & $\begin{array}{l}\text { C. Poverpivot in BI is implemented in in SQL Server } \\
\text { 2008R2 }\end{array}$ \\
\hline $\begin{array}{l}\text { 4. Introduced geospatial data types with few common features in } \\
\text { ssrs2008 }\end{array}$ & $\begin{array}{l}\text { D. Additional feature added geospatial data types in } \\
\text { ssrs2008, including mapping, routing and custom shaps }\end{array}$ \\
\hline
\end{tabular}

Table 7. Difference between Sql server 2008 and 2012

\begin{tabular}{|l|l|}
\hline SQL Server $\mathbf{2 0 0 8}$ & SQL Server $\mathbf{2 0 1 2}$ \\
\hline 1.Code name & A. Code name Denali \\
\hline 2.Query page splitting is not there in sql2008 & B. Query page splitting is implemented in sql2012 \\
\hline 3. It can support only 1000 partitions & C. It can support upto 1500 partitions \\
\hline 4. String function concate and format are not available sql2008 & D. String function concate and format are available sql2012 \\
\hline 5. Analysis service dosen,t have BISM sql2008 & E. Analysis service include BISM sql2012 \\
\hline 6. Order by clause doesn,t have OFFSET/FETCH options & F. Order by clause with OFFSET/FETCH options \\
\hline
\end{tabular}

\section{DISTRIBUTION ,PARALLELISM AND BIG DATA IN SQL SERVER VERSIONS}

According the role of Distribution, Parallelism and Big Data in Microsoft SQL Server specifically reviewed[6].

Parallelism is a feature in SQL Server which allows expensive queries to utilize more threads in order to complete quicker. The query optimizer makes the determination of how expensive a query is based upon the Cost Threshold for Parallelism setting set at the SQL Server Instance level. This cost threshold for parallelism is an estimate (roughly defined in seconds) that the query optimizer has determined a statement will take based on an estimated (or cached) execution plan. Generally the queries that qualify for parallelism are high IO queries. In a normal process, a source is read using a single SPID (Server Process ID) and it output using the same SPID. In parallelism, multiple SPIDs are used to read a source (this is known as distributing streams), then an operation may be performed in the streams, then the streams are gathered[7].

Big data is a term for data sets that are so large or complex that traditional data processing applications are inadequate. Challenges include analysis, capture, data curation, search, sharing, storage, transfer, visualization, querying, updating and information privacy. The term often refers simply to the use of predictive analytics or certain other advanced data analytics methods that extract value from data, and seldom to a particular size of data set. Accuracy in big data may lead to more confident decision making, and better decisions can result in greater operational efficiency, cost reduction and reduced risk[8].

A distributed database is a database in which storage devices are not all attached to a common processing unit such as the $\mathrm{CPU}$, and which is controlled by a distributed database management system. It may be stored in multiple computers, located

in the same physical location; or may be dispersed over a network of interconnected computers. Unlike parallel systems, in which the processors are tightly coupled and constitute a single database system, a distributed database system consists of loosely coupled sites that share no physical components. The Distributor is a server that contains the distribution database, which stores metadata and history data for all types of replication and transactions for transactional replication. To set up replication, you must configure a Distributor. Each Publisher can be assigned to only a single Distributor instance, but multiple publishers can share a Distributor. The Distributor uses these additional resources on the server where it is located. 
4. DISTRIBUTION, PARALLELISM

AND BIG DATA FEATURES IN SQL

SERVER VERSIONS (DETAILS)[9]

4.1 The following tables to determine which

features are supported by the different

editions of SQL Server 2000

Table 8. SQL Server 2000 Database Engine Features

\begin{tabular}{|l|l|l|l|l|l|l|l|}
$\begin{array}{l}\text { Database } \\
\text { Engine } \\
\text { Feature }\end{array}$ & $\begin{array}{l}\text { Enterprise } \\
\text { Edition }\end{array}$ & $\begin{array}{l}\text { Standard } \\
\text { Edition }\end{array}$ & $\begin{array}{l}\text { Personal } \\
\text { Edition }\end{array}$ & $\begin{array}{l}\text { Developer } \\
\text { Edition }\end{array}$ & $\begin{array}{l}\text { Desktop } \\
\text { Engine } \\
\text { (MSDE } \\
\text { 2000) }\end{array}$ & $\begin{array}{l}\text { SQLServer } \\
\text { CE }\end{array}$ & $\begin{array}{l}\text { Enterprise } \\
\text { Evaluati } \\
\text { on Edition }\end{array}$ \\
\hline $\begin{array}{l}\text { Parallel } \\
\text { DBCC }\end{array}$ & Supported & N/A & N/A & Supported & N/A & N/A & Supported \\
\hline $\begin{array}{l}\text { Parallel } \\
\text { CREATE } \\
\text { INDEX }\end{array}$ & Supported & N/A & N/A & Supported & N/A & N/A & Supported \\
\hline $\begin{array}{l}\text { Distributed } \\
\begin{array}{l}\text { artitioned } \\
\text { Cubes }\end{array}\end{array}$ & Supported & N/A & N/A & Supported & N/A & N/A & Supported \\
\hline
\end{tabular}

\subsection{The following tables to determine which} features are supported by the different editions of SQL Server 2005.

The following abbreviations represent SQL Server 2005 editions

- $\quad \mathrm{EE}=\mathrm{SQL}$ Server 2005 Enterprise Edition
- $\quad \mathrm{DE}=\mathrm{SQL}$ Server 2005 Developer Edition

- $\quad \mathrm{SE}=\mathrm{SQL}$ Server 2005 Standard Edition

- $\mathrm{WG}=\mathrm{SQL}$ Server 2005 Workgroup Edition

- $\quad$ SSE $=$ SQL Server 2005 Express Edition

- SSEA = SQL Server 2005 Express Edition with Advanced Services

Table 9. SQL Server 2005 Database Engine Features

\begin{tabular}{|c|c|c|c|c|c|}
\hline $\begin{array}{l}\text { DatabaseEngine Features, } \\
\text { Enhancements }\end{array}$ & $\begin{array}{l}\text { EE(32bit) } \\
\text { EE(64bit) } \\
\text { DE(32bit) } \\
\text { DE(64bit) }\end{array}$ & $\begin{array}{l}\text { SE(32bit) } \\
\text { SE (64bit) }\end{array}$ & $\begin{array}{l}\text { WG } \\
\text { (32bit) }\end{array}$ & $\begin{array}{l}\text { SSE } \\
\text { (32bit) }\end{array}$ & $\begin{array}{l}\text { SSEA } \\
\text { (32bit) }\end{array}$ \\
\hline $\begin{array}{l}\text { Transact-SQL Enhancements, Distributed } \\
\text { Query }\end{array}$ & Yes & Yes & Yes & Yes & Yes \\
\hline $\begin{array}{l}\text { Manageability Features, Parallel Service } \\
\text { Security }\end{array}$ & Yes & No & No & No & No \\
\hline $\begin{array}{l}\text { Database Maintenance Features, Parallel } \\
\text { Index Operations }\end{array}$ & Yes & No & No & No & No \\
\hline $\begin{array}{l}\text { Scalability and Performance Features, } \\
\text { Parallel DBCC }\end{array}$ & Yes & No & No & No & No \\
\hline $\begin{array}{l}\text { Scalability and Performance Features, } \\
\text { Updateable Distributed Partitioned } \\
\text { Views }\end{array}$ & Yes & No & No & No & No \\
\hline
\end{tabular}

Table 10. SQL Server 2005 Analysis Services Features

\begin{tabular}{|l|c|c|c|c|c|}
\hline $\begin{array}{l}\text { Analysis Services Features, } \\
\text { Enhancements }\end{array}$ & $\begin{array}{l}\text { EE(32bit) } \\
\text { EE(64bit) } \\
\text { DE(32bit) } \\
\text { DE(64bit) }\end{array}$ & $\begin{array}{c}\text { SE } \\
\text { (32bit) }\end{array}$ & $\begin{array}{l}\text { WG } \\
\text { (32bit) }\end{array}$ & $\begin{array}{l}\text { SSE } \\
\text { (32bit) } \\
\text { SSEA } \\
\text { (32bit) }\end{array}$ & $\begin{array}{l}\text { SE } \\
\text { (64bit) }\end{array}$ \\
\hline $\begin{array}{l}\text { Scalability and Performance Features, } \\
\text { Auto Parallel Partition Processing }\end{array}$ & Yes & No & No & No & No \\
\hline $\begin{array}{l}\text { Scalability and Performance Features, } \\
\text { Distributed Partitioned Cubes }\end{array}$ & Yes & No & No & No & No \\
\hline Data Mining Features , Parallelism for & Yes & No & No & No & No \\
\hline
\end{tabular}




\begin{tabular}{|l|c|c|c|c|c|}
\hline model processing & & & & \\
\hline $\begin{array}{l}\text { Data Mining Features , Parallelism for } \\
\text { model prediction }\end{array}$ & Yes & No & No & No & No \\
\hline
\end{tabular}

Table 11. SQL Server 2005 Notification Services Features

\begin{tabular}{|c|c|c|c|c|c|}
\hline $\begin{array}{l}\text { Notification Services Features, } \\
\text { Enhancements }\end{array}$ & $\begin{array}{l}\text { EE(32bit) } \\
\text { EE(64bit) } \\
\text { DE(32bit) } \\
\text { DE(64bit) }\end{array}$ & $\begin{array}{c}\text { SE } \\
\text { (32bit) }\end{array}$ & $\begin{array}{l}\text { WG } \\
\text { (32bit) }\end{array}$ & $\begin{array}{l}\text { SSE } \\
\text { (32bit) } \\
\text { SSEA } \\
\text { (32bit) }\end{array}$ & $\begin{array}{l}\text { SE } \\
\text { (64bit) }\end{array}$ \\
\hline $\begin{array}{lll}\text { Scalability Features, Parallelism } & \text { and } \\
\text { Multicast and Distributed deployment } & \end{array}$ & Yes & No & No & No & No \\
\hline
\end{tabular}

\subsection{The following tables to determine which} features are supported by the different editions of SQL Server 2008.

Table 12. Scalability

\begin{tabular}{|l|l|l|l|l|l|l|}
\hline Feature Name & Enterprise & Standard & Workgroup & Web Express & Express Tools & Express Advanced \\
\hline Partition able & Yes & & & & & \\
\hline parallelism & Yes & & & & & \\
\hline
\end{tabular}

Table 13. Manageability

\begin{tabular}{|l|l|l|l|l|l|l|}
\hline Feature Name & Enterprise & Standard & Workgroup & Web Express & Express Tools & Express Advanced \\
\hline $\begin{array}{l}\text { Distributed } \\
\text { partitioned } \\
\text { views }\end{array}$ & Yes & $\begin{array}{l}\text { Partial. } \\
\text { Distributed } \\
\text { Partitioned } \\
\text { Views are not } \\
\text { updatable }\end{array}$ & $\begin{array}{l}\text { Partial. } \\
\text { Distributed } \\
\text { Partitioned } \\
\text { Views are not } \\
\text { updatable }\end{array}$ & $\begin{array}{l}\text { Partial. } \\
\text { Distributed } \\
\text { Partitioned } \\
\text { Views are not } \\
\text { updatable }\end{array}$ & $\begin{array}{l}\text { Partial. } \\
\text { Distributed } \\
\text { Partitioned } \\
\text { Views not not } \\
\text { updatable }\end{array}$ & $\begin{array}{l}\text { Partial. Distributed } \\
\text { Partitioned Views } \\
\text { are not updatable }\end{array}$ \\
\hline $\begin{array}{l}\text { Parallel index } \\
\text { operations }\end{array}$ & Yes & & & & & \\
\hline $\begin{array}{l}\text { Parallel database } \\
\text { backup } \\
\text { checksum check }\end{array}$ & Yes & & & & & \\
\hline
\end{tabular}

Table 14. Data Warehouse Scale and Performance

\begin{tabular}{|l|l|l|l|l|l|l|}
\hline Feature Name & Enterprise & Standard & Workgroup & $\begin{array}{l}\text { Web } \\
\text { Express }\end{array}$ & $\begin{array}{l}\text { Express } \\
\text { Tools }\end{array}$ & $\begin{array}{l}\text { Express } \\
\text { Advanced }\end{array}$ \\
\hline $\begin{array}{l}\text { Auto parallel partition } \\
\text { processing }\end{array}$ & Yes & & & & \\
\hline $\begin{array}{l}\text { Distributed partitioned } \\
\text { cubes }\end{array}$ & Yes & & & & & \\
\hline
\end{tabular}

Table 15. Data Mining

\begin{tabular}{|l|l|l|l|l|l|l|}
\hline Feature Name & Enterprise & Standard & Workgroup & $\begin{array}{l}\text { Web } \\
\text { Express }\end{array}$ & $\begin{array}{l}\text { Express } \\
\text { Tools }\end{array}$ & $\begin{array}{l}\text { Express } \\
\text { Advanced }\end{array}$ \\
\hline Parallel model processing & Yes & & & & & \\
\hline
\end{tabular}




\subsection{The following tables to determine which \\ features are supported by the different editions of SQL Server 2008 R2.}

Table 16. Scalability and Performance

\begin{tabular}{|c|c|c|c|c|c|c|}
\hline Feature Name & Datacenter & Standard & Workgroup & $\begin{array}{l}\text { Express with } \\
\text { Advanced } \\
\text { Services } \\
\end{array}$ & $\begin{array}{l}\text { Express } \\
\text { with Tools }\end{array}$ & Express \\
\hline Parallel index operations & Yes & Yes & & & & \\
\hline $\begin{array}{l}\text { Parallel consistency checks } \\
\text { (DBCC) }\end{array}$ & Yes & Yes & & & & \\
\hline $\begin{array}{l}\text { Distributed Partitioned } \\
\text { Views }\end{array}$ & Yes & Yes & $\begin{array}{l}\text { Partial. } \\
\text { Distributed } \\
\text { Partitioned } \\
\text { Views are not } \\
\text { updatable }\end{array}$ & $\begin{array}{l}\text { Partial. } \\
\text { Distributed } \\
\text { Partitioned } \\
\text { Views are not } \\
\text { updatable }\end{array}$ & $\begin{array}{l}\text { Partial. } \\
\text { Distributed } \\
\text { Partitioned } \\
\text { Views are } \\
\text { not } \\
\text { updatable }\end{array}$ & $\begin{array}{l}\text { Partial. } \\
\text { Distributed } \\
\text { Partitioned Views } \\
\text { are not updatable }\end{array}$ \\
\hline
\end{tabular}

Table 17. Data Warehouse

\begin{tabular}{|l|l|l|l|l|l|l|}
\hline Feature Name & Datacenter & Standard & Workgroup & $\begin{array}{l}\text { Express with } \\
\text { Advanced } \\
\text { Services }\end{array}$ & $\begin{array}{l}\text { Express } \\
\text { with Tools }\end{array}$ & Express \\
\hline $\begin{array}{l}\text { Parallel query processing } \\
\text { on partitioned tables and } \\
\text { indices }\end{array}$ & Yes & Yes & & & \\
\hline $\begin{array}{l}\text { Distributed partitioned } \\
\text { cubes }\end{array}$ & Yes & Yes & & & & \\
\hline
\end{tabular}

Table 18 . Analysis Services - Advanced Analytic Functions

\begin{tabular}{|l|l|l|l|l|l|l|}
\hline Feature Name & Datacenter & Standard & Workgroup & $\begin{array}{l}\text { Express with } \\
\text { Advanced } \\
\text { Services }\end{array}$ & $\begin{array}{l}\text { Express } \\
\text { with Tools }\end{array}$ & Express \\
\hline $\begin{array}{l}\text { Partitioned cubes and } \\
\text { distributed partitioned } \\
\text { cubes }\end{array}$ & Yes & Yes & & & \\
\hline
\end{tabular}

Table 19. Data Mining

\begin{tabular}{|l|l|l|l|l|l|l|}
\hline Feature Name & Datacenter & Standard & Workgroup & $\begin{array}{l}\text { Express with } \\
\text { Advanced } \\
\text { Services }\end{array}$ & $\begin{array}{l}\text { Express } \\
\text { with Tools }\end{array}$ & Express \\
\hline Parallel model processing & Yes & Yes & & & & \\
\hline
\end{tabular}

\subsection{This topic provides details of features supported by the different editions of SQL Server 2012.}

Table 20. Scalability and Performance

\begin{tabular}{|l|l|l|l|l|l|l|}
\hline Feature Name & $\begin{array}{l}\text { Business } \\
\text { Intelligence }\end{array}$ & $\begin{array}{l}\text { Standard } \\
\text { web }\end{array}$ & Workgroup & $\begin{array}{l}\text { Express with } \\
\text { Advanced } \\
\text { Services }\end{array}$ & $\begin{array}{l}\text { Express } \\
\text { with Tools }\end{array}$ & Express \\
\hline Partition Table Parallelism & Yes & & & & \\
\hline
\end{tabular}

Table 21. Management Tools

\begin{tabular}{|l|l|l|l|l|l|l|}
\hline Feature Name & $\begin{array}{l}\text { Business } \\
\text { Intelligence }\end{array}$ & $\begin{array}{l}\text { Standard } \\
\text { web }\end{array}$ & Workgroup & $\begin{array}{l}\text { Express with } \\
\text { Advanced } \\
\text { Services }\end{array}$ & $\begin{array}{l}\text { Express } \\
\text { with Tools }\end{array}$ & Express \\
\hline
\end{tabular}




\begin{tabular}{|l|l|l|l|l|l|l|}
\hline $\begin{array}{l}\text { Distributed Replay Admin } \\
\text { Tool }\end{array}$ & Yes & Yes & Yes & Yes & Yes & Yes \\
\hline Distributed Replay Client & Yes & No & Yes & Yes & & \\
\hline $\begin{array}{l}\text { Distributed Replay } \\
\text { Controller }\end{array}$ & Yes & No & Yes & Yes & & \\
\hline
\end{tabular}

Table 22. RDBMS Manageability

\begin{tabular}{|l|l|l|l|l|l|l|}
\hline Feature Name & $\begin{array}{l}\text { Business } \\
\text { Intelligence }\end{array}$ & $\begin{array}{l}\text { Standard } \\
\text { web }\end{array}$ & Workgroup & $\begin{array}{l}\text { Express with } \\
\text { Advanced } \\
\text { Services }\end{array}$ & $\begin{array}{l}\text { Express } \\
\text { with Tools }\end{array}$ & Express \\
\hline $\begin{array}{l}\text { Distributed partitioned } \\
\text { views }\end{array}$ & Yes & $\begin{array}{l}\text { Partial. } \\
\text { Distributed } \\
\text { Partitioned } \\
\text { Views are } \\
\text { not } \\
\text { updatable }\end{array}$ & $\begin{array}{l}\text { Partial. } \\
\text { Distributed } \\
\text { Partitioned } \\
\text { Views are not } \\
\text { updatable }\end{array}$ & $\begin{array}{l}\text { Partial. } \\
\text { Distributed } \\
\text { Partitioned } \\
\text { Views are not } \\
\text { updatable }\end{array}$ & $\begin{array}{l}\text { Partial. } \\
\text { Distributed } \\
\text { Partitioned } \\
\text { Views not are } \\
\text { updatable }\end{array}$ & $\begin{array}{l}\text { Partial. } \\
\text { Distributed } \\
\text { Partitioned } \\
\text { Views are not } \\
\text { updatable }\end{array}$ \\
\hline Parallel indexed operations & Yes & & & & & \\
\hline Parallel consistency check & Yes & & & & \\
\hline
\end{tabular}

Table 23. Data Warehouse

\begin{tabular}{|l|l|l|l|l|l|l|}
\hline Feature Name & $\begin{array}{l}\text { Business } \\
\text { Intelligence }\end{array}$ & Standard web & Workgroup & $\begin{array}{l}\text { Express with } \\
\text { Advanced } \\
\text { Services }\end{array}$ & $\begin{array}{l}\text { Express with } \\
\text { Tools }\end{array}$ & Express \\
\hline $\begin{array}{l}\text { Parallel query } \\
\text { processing on } \\
\text { partitioned tables } \\
\text { and indices }\end{array}$ & Yes & & & & & \\
\hline
\end{tabular}

Table 24. Data Mining

\begin{tabular}{|l|l|l|l|l|l|l|}
\hline Feature Name & $\begin{array}{l}\text { Business } \\
\text { Intelligence }\end{array}$ & Standard web & Workgroup & $\begin{array}{l}\text { Express with } \\
\text { Advanced } \\
\text { Services }\end{array}$ & $\begin{array}{l}\text { Express with } \\
\text { Tools }\end{array}$ & Express \\
\hline $\begin{array}{l}\text { Parallel Model } \\
\text { Processing }\end{array}$ & Yes & Yes & & & \\
\hline
\end{tabular}

\subsection{This topic provides details of features supported by the different editions of SQL Server 2014}

Table 25. Scalability and Performance

\begin{tabular}{|l|l|l|l|l|l|l|}
\hline Feature Name & $\begin{array}{l}\text { Business } \\
\text { Intelligence }\end{array}$ & Standard web & Workgroup & $\begin{array}{l}\text { Express with } \\
\text { Advanced } \\
\text { Services }\end{array}$ & $\begin{array}{l}\text { Express with } \\
\text { Tools }\end{array}$ & Express \\
\hline $\begin{array}{l}\text { Partition Table } \\
\text { Parallelism }\end{array}$ & Yes & & & & \\
\hline
\end{tabular}

Table 26. Management Tools

\begin{tabular}{|l|l|l|l|l|l|l|}
\hline Feature Name & $\begin{array}{l}\text { Business } \\
\text { Intelligence }\end{array}$ & Standard web & Workgroup & $\begin{array}{l}\text { Express with } \\
\text { Advanced } \\
\text { Services }\end{array}$ & $\begin{array}{l}\text { Express with } \\
\text { Tools }\end{array}$ & Express \\
\hline $\begin{array}{l}\text { Distributed Replay } \\
\text { Admin Tool }\end{array}$ & Yes & Yes & Yes & Yes & Yes & Yes \\
\hline $\begin{array}{l}\text { Distributed Replay } \\
\text { Client }\end{array}$ & Yes & No & Yes & Yes & & \\
\hline $\begin{array}{l}\text { Distributed Replay } \\
\text { Controller }\end{array}$ & Yes & No & Yes & Yes & & \\
\hline
\end{tabular}


Table 27. RDBMS Manageability

\begin{tabular}{|l|l|l|l|l|l|l|}
\hline Feature Name & $\begin{array}{l}\text { Business } \\
\text { Intelligence }\end{array}$ & Standard web & Workgroup & $\begin{array}{l}\text { Express with } \\
\text { Advanced } \\
\text { Services }\end{array}$ & $\begin{array}{l}\text { Express with } \\
\text { Tools }\end{array}$ & Express \\
\hline $\begin{array}{l}\text { Distributed } \\
\text { partitioned views }\end{array}$ & Yes & $\begin{array}{l}\text { Partial. } \\
\text { Distributed } \\
\text { Partitioned } \\
\text { Views are not } \\
\text { updatable } \\
\text { Indexed }\end{array}$ & $\begin{array}{l}\text { Partial. } \\
\text { Distributed } \\
\text { Partitioned } \\
\text { Views are not } \\
\text { updatable }\end{array}$ & $\begin{array}{l}\text { Distributed } \\
\text { Partitioned } \\
\text { Views are not } \\
\text { updatable }\end{array}$ & $\begin{array}{l}\text { Partial. } \\
\text { Distributed } \\
\text { Partitioned } \\
\text { Views are not } \\
\text { updatable }\end{array}$ & $\begin{array}{l}\text { Partial. } \\
\text { Distributed } \\
\text { Partitioned } \\
\text { Views are not } \\
\text { updatable }\end{array}$ \\
\hline $\begin{array}{l}\text { Parallel } \\
\text { operations }\end{array}$ & Yes & & & & & \\
\hline $\begin{array}{l}\text { Parallel consistency } \\
\text { check }\end{array}$ & Yes & & & & \\
\hline
\end{tabular}

Table 28. Data Warehouse

\begin{tabular}{|l|l|l|l|l|l|l|}
\hline Feature Name & $\begin{array}{l}\text { Business } \\
\text { Intelligence }\end{array}$ & Standard web & Workgroup & $\begin{array}{l}\text { Express with } \\
\text { Advanced } \\
\text { Services }\end{array}$ & $\begin{array}{l}\text { Express with } \\
\text { Tools }\end{array}$ & Express \\
\hline $\begin{array}{l}\text { Parallel query } \\
\text { processing on } \\
\text { partitioned tables } \\
\text { and indices }\end{array}$ & Yes & & & & & \\
\hline
\end{tabular}

Table 29. Data Mining

\begin{tabular}{|l|l|l|l|l|l|l|}
\hline Feature Name & $\begin{array}{l}\text { Business } \\
\text { Intelligence }\end{array}$ & Standard web & Workgroup & $\begin{array}{l}\text { Express with } \\
\text { Advanced } \\
\text { Services }\end{array}$ & $\begin{array}{l}\text { Express with } \\
\text { Tools }\end{array}$ & Express \\
\hline $\begin{array}{l}\text { Parallel Model } \\
\text { Processing }\end{array}$ & Yes & Yes & & & & \\
\hline
\end{tabular}

\section{CONCLUSION}

According the role of Distribution Parallelism and Big Data in information technology world, Given the role it is worth More studying. Parallelism is a feature in SQL Server which allows expensive queries to utilize more threads in order to complete quicker. Big data is a term for data sets that are so large or complex that traditional data processing applications are inadequate. A distributed database is a database in which storage devices are not all

attached to a common processing unit such as the CPU, and which is controlled by a distributed database management system. Given the importance of the need for more research.

There for Distribution, Parallelism and Big Data in SQL Server need for strong research for advance database works.

\section{REFERENCES}

[1] www.databasejournal.com/features/article.php/3593466/ MS-SQL-Series.htm

[2] https://msdn.microsoft.com/en-us/library/SQL Server Books http://www.databasejournal.com/features/mssq1/10-newfeatures-worth-exploring-in-sql-server-2016.html

[3] https://technet.microsoft.com/enus/library/mt590198(v=sql.1).aspx

[4] Laden Sie die Testversion herunter: Microsoft SQL Server 2014" (in German). Microsoft. Retrieved 2014$11-05$

[5] Database System | Performance \& Scalability | SQL Server 2012 Business Intelligence Editions". Microsoft.com. Retrieved 2013-06-15.

[6] SQL Server 2014 Service Pack 1 release information". Microsoft.com. Microsoft, Inc. Retrieved 17 May 2015

[7] SQL Server 2012 Express LocalDB". msdn.microsoft.com. Retrieved March 11, 2013.

[8] SQL Server 2012-2014-Explore. Server Cloud. Microsoft.com (2013-04-17). Retrieved on 2014-03-23. 